\title{
TNFAIP8 inhibits the proliferation of gastric cancer cells by inhibiting mTOR-Akt-ULK1 signal pathway and activating autophagy
}

\section{Zheng Chen}

Shandong Provincial Hospital Affiliated to Shandong First Medical University

Jianguo Zhang

Liaocheng Dongchangfu People's Hospital

\section{Chenyang Dong}

Shandong Provincial Hospital Affiliated to Shandong First Medical University

\section{Dongsheng Li}

Shandong Provincial Hospital Affiliated to Shandong First Medical University

\section{Yuehan Yin}

Shandong Provincial Hospital Affiliated to Shandong First Medical University

\section{Wenhai Yu}

Shandong Provincial Hospital Affiliated to Shandong First Medical University

\section{Yuezhi Chen ( $\nabla$ chenyuezhi@126.com )}

Shandong Provincial Hospital Affiliated to Shandong First Medical University https://orcid.org/00000003-4226-4939

\section{Primary research}

Keywords: TNFAIP8, Gastric cancer, mTOR-Akt-ULK1, Autophagy

Posted Date: August 29th, 2020

DOI: https://doi.org/10.21203/rs.3.rs-21653/v3

License: (1) (i) This work is licensed under a Creative Commons Attribution 4.0 International License. Read Full License 


\section{Abstract}

Background : The purpose of this article was to study the role of TNFAIP8 in gastric cancer. Methods : RTPCR was used to detect the expression of TNFAIP8 mRNA and protein level in normal gastric mucosa cells and four gastric cancer cell lines. TNFAIP8 was silenced or overexpressed in two cell lines, CCK-8 cell viability was used, transwell experiment was used to detect cell invasion capability, and flow cytometry was used to detect cell apoptosis. TNFAIP was silenced or overexpressed in a cell line, and nude mice were inoculated to form transplanted tumors. HE staining and immunohistochemistry staining were used to detect the histopathological changes of tumors. Results: The mRNA and protein expression of TNFAIP8 was significantly up-regulated in GC patients and cells. After silencing and overexpressing TNFAIP8, gastric cancer cells with high expression increased, apoptosis decreased, and cell invasion increased. Expression of mTOR-Akt-ULK1 signal pathway was inhibited and autophagy signal was activated. Conclusions: Our findings indicate that TNFAIP8 inhibited gastric cancer cells by inhibiting mTOR-Akt-ULK1 signal pathway and activating autophagy signal.

\section{Background}

Gastric cancer (GC) is one of the most common and fatal malignant tumors in the world. It is difficult to treat, and it is easy to metastasize and spread. At the same time, its poor prognosis is related to lymph node and peritoneal metastasis of gastric cancer, which is the main cause of recurrence of GC[1]. According to statistics, the number of new gastric cancers and cancer deaths in the world in 2020 reached about 14.1 million and 8.2 million respectively[2]. Most of these cases occurred in developing countries. GC, as a common malignant tumor in digestive system, posed a serious threat to human health like liver cancer and colorectal cancer. Although its incidence rate has decreased in the past few years, its death rate was still the second highest among all kinds of malignant tumors[3]. Among the existing treatment methods, although surgical resection and adjuvant therapy have made great progress, and even some GC could be cured in the early stage, unfortunately most patients were found to be in the late stage, and the originally effective treatment methods have become ineffective or ineffective[4]. Therefore, it has become an urgent need for clinical workers and laboratory researchers to find an effective tumor marker for early diagnosis and improvement of therapeutic effects.

A new candidate oncogene, tumor necrosis factor-a-induced protein 8 (TNF-AIP8) has gradually attracted the attention of scholars and extensive attention[5]. Members of this family include TNFAIP8, TNFAIP1 (TNF-AINDUCEDPLOTEIN 8-LIKE 1, TIPEL), TIPE2 and TIPE3F7L[6]. Although they have high homology, their biological behaviors was proved to be different in related experiments of Lord W. Among them, TNFAIP 8 is also called GG2-1, SCC-S2, MDC-3.13, which is the first subtype found in this family[7]. The gene is located on chromosome $5 q 23.1$ and is expressed in most malignant foot tumor tissues. Studies on these tumors have all suggested that signal transduction pathway affects many processes such as cell apoptosis, and therefore plays an important role in the formation and development of tumors[8]. In recent years, a series of functions of TNFAIP 8 in tumor formation have been continuously confirmed. These results showed that TNFAIP 8 played an important role in the occurrence and development of 
tumors and was one of the important components in regulating cell apoptosis[9]. It was involved in the regulation of cell proliferation in different types of tumors, affecting invasion, migration, apoptosis and drug resistance. Previous studies have found that TNFAIP 8 expression could be detected in most malignant tumor tissues such as breast cancer, colon cancer, ovarian epithelial cell cancer, prostate cancer $[6,10,11]$. However, there are few reports on its research in GC and its mechanism is even less. The purpose of this article is to study its role in GC.

\section{Materials And Methods}

\section{Reagents}

RPMI-1640 culture solution was purchased from Gibco Company of the United States, fetal bovine serum and double antibody were both purchased from Thermophilic Technology Company. CCK-8 was purchased from Japan Tongren Chemical Company. Transwell chamber and artificial basement membrane were purchased from BD company in USA. The flow detection kit was purchased from Nanjing Keygen Biology Co., Ltd. LC-31/LC-32 (\#12741,1:1000), P62 (\#23214,1:1000), p-Akt (\#4060, Ser 473,1:1000), Akt (\#4685), p-mTOR (\#5536, Ser2448,1:1000), p-ULK1 (\#14202,Ser757,1:1000), ULK1 (\#6439,1:1000), GAPDH (\#5147,1:1000) antibodies were purchased from Cell Signaling Technology. TNFAIP8 (\#ab251212) antibody was purchased from Abcam.

\section{Collection of gastric cancer tissues and normal tissues}

This study was examined by the Ethics Committee of Shandong Provincial Hospital Affiliated to Shandong First Medical University (KY2018018) before the start of the experiment, and was registered in the clinical laboratory center of China (CHiCTR1800017378). 1417 patients (708 men [50\%], 709 women [46\%]).

\section{Animals}

24 BALB/C nu/nu male nude mice were purchased from Shanghai Sciple-Bikai Experimental Animal Co., Ltd. with the quality certificate number of SCXK $(\mathrm{Hu})$ 2019-0016. The mice were 4 weeks old, weighing 15 $\mathrm{g}$, and were raised under SPF conditions. All operations were carried out in accordance with the animal ethics regulations of Shandong Provincial Hospital Affiliated to Shandong First Medical University.

\section{Cell culture}

GES-1, SGC-7901, NCI-N87, MKN-28, MGC-803 were purchased from North Carolina Cell Resource Center (USA) and placed in RPMI- 1640 culture solution containing $10 \%$ fetal bovine serum, and cultured in $37^{\circ} \mathrm{C}$, $5 \% \mathrm{CO}_{2}$ incubator. When its adherent growth reached $70 \%-80 \%$ confluence, it was digested and subcultured with $0.05 \%$ pancreatin.

RT-qPCR detection of TNFAIP8 gene expression in several gastric cells 
GES-1, SGC-7901, NCI-N87, MKN-28, MGC-803 cells were cultured and collected to detect TNFAIP8 expression. Cell RNA was extracted according to Trizol reagent instructions and cDNA was synthesized according to cDNA reverse transcription kits instructions. RT-qPCR reaction was carried out on StepOnePlus real-time fluorescence quantitative PCR system by two-step method. The reaction conditions were: pre-denaturation at $95^{\circ} \mathrm{C}$ for $95^{\circ} \mathrm{C} 1 \mathrm{~min}$, denaturation at $95^{\circ} \mathrm{C}$ for $5 \mathrm{~s}$, annealing and extension at $60^{\circ} \mathrm{C}$ for $60^{\circ} \mathrm{C} 30 \mathrm{~S}$, with a total of 40 cycles.

\section{Cell viability}

Gastric cancer cells that were silent or overexpressed TNFAIP8 were inoculated into 96-well plates with 5,000 cells per well. One 96 -well plate was taken out after 24,48 and $72 \mathrm{~h}$ of conventional culture. $10 \mathrm{uL}$ of CCK-8 solution $(5 \mathrm{mg} / \mathrm{m} 1)$ was added to each well and the culture was continued for $4 \mathrm{~h} .150$ mountains of DMSO solution was added to each well, and then were shake on a shaker at low speed for $10 \mathrm{~min}$. At last, it was detected at $490 \mathrm{~nm}$ on the microplate reader.

\section{Establishment of human gastric cancer model in nude mice}

First, nude mice were adaptively fed for 1 week, and human gastric cancer MKN-28 cells which were silent in logarithmic growth phase and overexpressed TNFAIP8 gene after pancreatin digestion were collected, centrifuged at $800 \mathrm{rpm}$ for 4 minutes, and the supernatant was discarded. It was made into a single cell suspension with a concentration of $2 \times 10^{7}$ cells $/ \mathrm{ml}$. In a sterile environment, $0.2 \mathrm{ml} /$ nude mouse was inoculated under the armpit, and the nude mouse was put back into the cage to continue feeding and observe the state of the nude mouse. A week later, 24 nude mice showed subcutaneous nodules of about $5 \mathrm{~mm}$, the model of human gastric cancer was established.

\section{Cell transfection}

MKN-45 cells were seeded into 6-well plates 1 day before transfection. Transfection was prepared when adherent cells reached a fusion degree of $40 \%$ to $60 \%$. The original medium was removed during transfection and washed with RPMI1640 medium (serum-free) 2 times. $4 \mu \mathrm{L}$ of lipofectamine 2000 liposomes was added to $500 \mu \mathrm{L}$ of serum-free RPMl 1640 culture solution, while $10 \mu \mathrm{L}$ of TNFAIP8 mimics storage solution was added to $500 \mu \mathrm{L}$ of serum-free RPMl1640 medium, and allowed to stand at room temperature for 5 minutes, and then the two were mixed and allowed to stand at room temperature for 20 minutes. The mixed solution was added to the cells, and the final concentration of mimics was 80 $\mathrm{nmol} / \mathrm{L}$. After 6 hours, the DMEM medium containing 10\% fetal bovine serum was replaced, and the culture was continued to be expanded for subsequent experiments. RNA sequence of TNFAIP8 was: forward, 5'-T C C A T C G C C A C C A C C T T A-3' and reverse, 5'-C T C T G C C T C C T T C T T G T T T T3'; GAPDH forward, 5'-G G C A A A T T C A A C G G C A C A G T C A-3' and reverse, 5'-G T C T C G C T C C T G G A A G A T G G T G A T-3'.

\section{Transwell experiment}


The small chamber was put into a culture plate, $300 \mu \mathrm{l}$ of pre-heated serum-free culture medium was added into the upper chamber, and the upper chamber was left to stand for 15-30 min at room temperature to rehydrate the matrix glue; The remaining culture solution was sucked off, and the cells to be tested in each group were made into a cell suspension of $5 \times 10^{5}$ cells $/ \mathrm{ml}$ using a serum-free medium containing bovine serum albumin (BSA). $100-200 \mu \mathrm{L}$ of cell suspension was taken and added into Transwell upper chamber, and $500 \mu \mathrm{l}$ of culture medium containing $20 \%$ FBS or chemokines was added into lower chamber; The cells at the bottom of the lower chamber were stained with $0.5 \%$ crystal violet and the cells at the inner side of the upper chamber were removed with cotton swabs. Finally, cell morphology was observed under microscope and cell numbers was counted.

\section{Flow cytometry}

Cell lines stably transferring TNFAIP8, stably transferring empty carriers and non-transferring cell lines are inoculated on a 96-well plate according to a ratio of $2 \times 10^{4} /$ well, each group of cells are collected and operated according to an instruction book of an apoptosis detection reagent kit, and the cells are detected by an up-flow cytometer immediately after the color was dyed. In this experimental study, in order to investigate the effect of mTOR-Akt-ULK1 signaling pathway on apoptosis, PI3K inhibitor 3Methyladenine(3-MA,10uM, dissolved in DMSO) and MTOR kinase activator 3-benzyl-5-((2-nitrophenoxy) methyl)-dihydrofuran-2 were added in the experiment. Annexin $V$ staining positive cells were early apoptotic cells and PI staining positive cells were necrotic cells, and anexin V and PI staining were double positive. The cells were late apoptotic cells, and anexin V and PI staining were double negative.

\section{Ki-67 staining}

Tumor tissues were fixed with $4 \%$ formaldehyde and embedded in paraffin. All tissues were cut into $4 \mu \mathrm{m}$ sections using a German thermoscience cryostat, and then tumor sections were incubated with primary Ki-67 antibody, sections were washed with PBS, secondary antibody were incubated according to the manufacturer's instructions.

\section{Western blotting}

The samples were minced and homogenized in ice-cold RIPA buffer containing $2 \mathrm{mM} \mathrm{PMSF}$. Then samples were centrifugated at $12000 \mathrm{~g}$ for $15 \mathrm{~min}$ at $4^{\circ} \mathrm{C}$. The total protein concentration was measured by the BCA assay kit. The proteins were subjected to SDS-PAGE electrophoresis, transferred to PVDF membranes, blocked with $5 \%$ skim dried milk for $2 \mathrm{~h}$, and then probed with primary antibodies at $4{ }^{\circ} \mathrm{C}$ overnight. After that, it was incubated with HRP-conjugated secondary antibody for $2 \mathrm{~h}$. The samples were visualized by an enhanced chemiluminescence (ECL) advanced kit and a gel imaging system (Tanon Science \& Technology Co., Ltd., China).

\section{Statistical analysis}


All data were presented as mean \pm SD. The statistical significance of differences between the means of each group was analyzed by one-way ANOVA followed by Tukey multiple comparison tests. For comparing two groups, Student's $t$ test was used. A p value less than 0.05 was considered statistically significant.

\section{Results}

TNFAIP8 gene expression and TNFAIP8 protein level in gastric mucosal cells and four types of gastric cancer cells

Compared with normal tissues, the expression of TNFAIP8 gene and TNFAIP8 protein level was higher in gastric cancer tissues (Fig.1A and 1B), In addition, compared with normal gastric mucosa GES-1 cells, the expression of TNFAIP8 gene in four gastric cancer cells SGC-7901, NCI-N87, MKN-28, and MGC-803 was significantly increased (Fig.1C).

Overexpression of TNFAIP8 gene increased cell viability, reduced apoptotic rate and increased migration ability

As shown in Figure 2A, in MKN-28 and MGC-803 cells, the silencing and over-expression of the TNFAIP8 gene was verified by PCR technology. Overexpression of TNFAIP8 gene obviously increased cell viability, reduced apoptotic rate and increased migration ability. In contrast, silent TNFAIP8 gene obviously genes obviously decreased cell viability, increased apoptotic rate and decreased migration ability (Fig. 2B, 2C, 2D).

Overexpression of TNFAIP8 gene increased tumor volume and tumor weight in transplanted tumor mice

Compared with normal cell transplanted tumor mice, over-expressing TNFAIP8 gene increased tumor volume and tumor quality in transplanted tumor mice, and silencing TNFAIP8 gene reduced tumor volume and tumor quality in transplanted tumor mice (Fig. 3A-3C). Ki67 immunohistochemical results showed that the number of positive cells with over-expressed TNFAIP8 gene was significantly reduced, and the number of positive cells with silenced TNFAIP8 gene was significantly reduced (Fig .3D).

\section{Overexpression of TNFAIP8 gene activated autophagy and inhibited mTOR-Akt-ULK1 signaling pathway}

As shown in Fig. 4, over-expression of the TNFAIP8 gene significantly increased the ratio of LC3I/LC-3II and P-ULK1 level and decreased the levels of P62, P-PI3K, and P-mTOR. In contrast, silent of the TNFAIP8 gene significantly decreased the ratio of LC3I/LC-3II and P-ULK1 level and increased the levels of P62, PPI3K, and P-mTOR.

Autophagy inhibitor and activator demonstrated the role of TNFAIP8 genes in regulating autophagy signals 
As shown in Fig. 5, over-expression of the TNFAIP8 gene significantly increased cell viability, decreased apoptotic rate and increased migration ability. However, autophagy inhibitor significantly reversed these changes, autophagy activator not only significantly reversed the above changes, but also reversed the changes of P-P3K, P-mTOR and p-ULK1 by overexpression of the TNFAIP8 gene (Fig.6).

\section{Discussion}

$\mathrm{GC}$, as one of the common malignant tumors of the digestive system, poses a serious threat to human health as well as smart cancer and colorectal cancer[12, 13]. In China, the current incidence of GC is 29.9 per 100,000 (male $41.3 / 100,000$ vs. female $18.5 / 100,000$ ), and the mortality rate is 22.3 per 100,000 (male 30.1/100,000). (VS. Women 14.6/100,000)[14, 15]. The current treatment method for GC is comprehensive surgery (including adjuvant chemotherapy, molecular report drug treatment) [16]. Although surgical resection and adjuvant chemotherapy have made great progress in the treatment of GC, some GC can be cured at an early stage $[17,18]$. Unfortunately, most patients are already in an incurable stage when they are discovered. Originally effective treatments have become ineffective or ineffective, and the overall prognosis is poor. The detection of EGF, cyclnE, p27, E-caerin, CD44v6, MMP-1, TIMP-1, HER-2, HER-3, and VEGF may have important significance for the individualized treatment of GC patients[19, 20]. However, the mechanism of gastric cancer has not been fully clarified so far, and there is no effective indicator for early diagnosis, early treatment, improved prognosis, and prolonged patient survival. Therefore, whether or not to find an effective tumor marker for early diagnosis and improve the treatment effect has become an urgent need for clinicians and laboratory researchers.

Recently, a new candidate oncogene, tumor necrosis factor-a-inducing protein 8 (Tumorcrosis factor-ainduce protein 8 , TNFAJP8), has gradually come into the sight of scholars and has attracted widespread attention [21, 22]. In recent years, a series of roles of TNFAIP8 in tumor formation have been continuously confirmed. These results showed that TNFAIP8 played a vital role in the development of tumors. It was involved in the regulation of cell proliferation in different tumor types, and tumor invasion, migration, death, and drug resistance. Xing et al. measured the expression of TNFAIP8 in non-small cell lung cancer tissues and adjacent normal lung tissues [23]. Miao et al. analyzed the expression pattern of TNFAIP8 in 92 colon cancer tissues by immunohistochemistry, and found that TNFAIP8 was overexpressed in 45 patients (48.9\%). Although TNFAIP8 plays a vital role in the genesis and development of tumors, the expression of TNFAIP8 in gastric cancer and its role in regulating the growth, invasion and migration of gastric cancer cells have not been determined. In our study, PCR results found that compared with normal tissues, the TNFAIP8 gene was significantly increased in gastric cancer tissues. In addition, compared with normal gastric mucosa tissue, TNFAIP8 genes were significantly increased in the four gastric cancer tissues. This result indicates that TNFAIP8 gene is involved in the pathological process of gastric cancer.

At present, there are few studies on the role of TNFAIP8 in gastric cancer, and it is difficult to determine whether down-regulation of TNFAIP8 will affect the biological characteristics of gastric cancer cells. In the first experiment, we have confirmed that TNFAIP8 was expressed in gastric cancer tissues and 
tissues, and its expression is higher than that of paracancerous tissue and normal gastric mucosal cells, suggesting that TNFAIP8 was closely related to the occurrence and development of gastric cancer.

At present, a type of cell death that does not depend on the apoptotic pathway-cell autophagy has become a research hotspot. On the one hand, autophagy can play an anti-tumor and anti-aging effect, but on the other hand, the autophagy process provided cells with nutrients to help them survive the harsh environment and also play a role in promoting the development of tumors[24]. It is a "double-edged sword" in the process of tumorigenesis and development. Hypoxia, ischemia, and radiation therapy can cause tumor cell autophagy to increase, tumor tissue lacks blood supply, and metastasis is prone to place tumor cells in a metabolic stress state[25]. In this study, over-expression of the TNFAIP8 significantly increased cell viability, decreased apoptotic rate and increased migration ability. However, autophagy inhibitor and activator significantly reversed these changes. The above phenomenon is very interesting, suggesting that the TNFAIP8 gene may have effect on tumor cells by activating autophagy signals and regulated the pathophysiology of gastric cancer.

Akt is a key molecule downstream of PI3K. It binds to PI3K through its N-terminal PH domain. At the same time, it can regulate the proliferation and survival of many types of cells [26]. Akt acts as a key regulator of multiple cell proliferation, differentiation and survival. mTOR is an important substrate of ATK, as a large protein with a C-terminal serine/threonine PI3K-related kinase domain. The mTOR signal pathway, as an important cell signal transduction pathway, is involved in physiological activities such as cell growth, survival, and autophagy. Studies have also shown that abnormal mTOR signals have profound effects on cell homeostasis, and may even lead to the development of pathological conditions, such as gastric cancer [27]. Tian et al used immunohistochemistry to detect the expression of AKT in tumor tissues and adjacent tissues of 128 patients with gastric cancer, and found that the expression level of AKT in tumor tissues was significantly higher than that in adjacent tissues and was related to the T stage[28]. The expression of phosphorylated AKT (phospho-AKT, p-AKT) was detected in tissue samples from 231 patients with gastric cancer. It was found that p-AKT was expressed in 119 samples (53\%). Statistical analysis showed that p-AKT Poor patient prognosis. Li et al. detected the expression of mTOR in 33 patients with GC and 30 healthy controls by immunohistochemistry, and found that the expression rate of mTOR in patients with GC was $51.5 \%$. There is almost no mTOR expression, suggesting that mTOR activation occurs during the occurrence and development of GC [29]. In addition, the study also found that mTOR expression is associated with late tumor stage, poor differentiation, and lymph node metastasis. ULK1 (Unc-51. Like. Autophage. Akinting. Kinase) complex initiates the formation of autosome. ULK1 could be regulated by mTORC1 and MAPK-related kinases. mTORC1 integrated growth factors, regulated oxygen content, amino acids and energy, and promote the synthesis of proteins related to cell growth metabolism[30]. When mTORC1 is activated, it reduced the activity of ULK1 kinase by phosphorylating ULK1 and ATG13. In this study, we investigated the relationship between the TNFAIP8 and the mTOR-Akt-ULK1signaling pathway and its role in gastric cancer. Our results showed that over-expressing TNFAIP8 significantly reduced p-Akt and p-mTOR levels and increased p-ULK1 level. In contrast, silenced TNFAIP8 significantly increased p-Akt and p-mTOR levels, and significantly reduced p-ULK1 level. Even more interesting was that when autophagy activators are added, these changes are 
significantly reversed. The above results showed that the TNFAIP8 inhibited the mTOR-Akt-ULK1 and involved in the physiological and pathological processes of gastric cancer.

\section{Conclusion}

In summary, this study found that the TNFAIP8 was highly expressed in gastric cancer tissues, and found that TNFAIP8 significantly inhibited the mTOR-Akt-ULK1 signaling pathway and activated autophagy signal. The above mechanisms may be involved in the physiological and pathological processes of gastric cancer and also provided a new target for treatment of gastric cancer.

\section{Abbreviations}

GC: Gastric cancer; TNF-AIP 8: Tumor necrosis factor-a-induced protein 8; ECL: Enhanced chemiluminescence; TIPE1: TNF-induceprotein8-lick1

\section{Declarations}

\section{Ethics approval and consent to participate}

All the experimental procedures were approved and executed in accordance with the Institutional Animal Care and Use Committee of Nanjing University of Chinese Medicine.

\section{Consent for publication}

Not applicable.

\section{Availability of data and material}

The data used to support the findings of this study are included in the article.

\section{Competing Interest}

There are no conflicts of interest to declare.

\section{Funding}

Not applicable.

\section{Authors' Contributions}

ZC, JGZ, CYD and YZC performed the experiments, analyzed the data and wrote the paper. DSL, YHY and WHY designed the present study and provided experimental materials. All authors read and approved the final manuscript.

\section{Acknowledgements}


Not applicable.

\section{References}

1. Ryun Park S: Management of gastric cancer: East vs West. Curr Prob/ Cancer 2015, 39(6):315-341.

2. Levi Z, Kark JD, Twig G, Katz L, Leiba A, Derazne E, Tzur D, Liphshitz I, Keinan-Boker L, Afek A: Body mass index at adolescence and risk of noncardia gastric cancer in a cohort of 1.79 million men and women. Cancer.

3. Medina Franco H: [Gastrointestinal oncology. Gastric cancer]. Revista de gastroenterologia de Mexico 2010, 75 Suppl 1:62-64.

4. Latif A: Gastric cancer. Update on diagnosis, staging, and therapy. Postgraduate medicine 1997, 102(4):231-236, 241.

5. Liu T, Jiang L, Yu L, Ge T, Wang J, Gao H: Association of TNFAIP8 gene polymorphisms with endometrial cancer in northern Chinese women. Cancer cell international 2019, 19:105.

6. Niture S, Dong X, Arthur E, Chimeh U, Niture SS, Zheng W: Oncogenic Role of Tumor Necrosis Factor a-Induced Protein 8 (TNFAIP8). 2018, 8(1).

7. Niture S, Moore J, Kumar D: TNFAIP8: Inflammation, Immunity and Human Diseases. Journal of cellular immunology 2019, 1(2):29-34.

8. Han Y, Tang Z, Zhao Y, Li Q, Wang E: TNFAIP8 regulates Hippo pathway through interacting with LATS1 to promote cell proliferation and invasion in lung cancer. 2018, 57(2):159-166.

9. Dong Q, Fu L, Zhao Y, Xie C, Li Q, Wang E: TNFAIP8 interacts with LATS1 and promotes aggressiveness through regulation of Hippo pathway in hepatocellular carcinoma. Oncotarget 2017, 8(9):15689-15703.

10. Xie Y, Zhou F, Zhao X: TNFAIP8 promotes cell growth by regulating the Hippo pathway in epithelial ovarian cancer. Experimental and therapeutic medicine 2018, 16(6):4975-4982.

11. Niture S, Ramalinga M, Kedir H, Patacsil D, Niture SS, Li J, Mani H, Suy S, Collins S, Kumar D: TNFAIP8 promotes prostate cancer cell survival by inducing autophagy. Oncotarget 2018, 9(42):26884-26899.

12. Sasaki S, Hiraiwa T, Ishimaru M, Funahashi K, Koike J, Seto Y, Yamashita H, Furushima K, Sakai K: [Multicentral Questionnaire Results for Consciousness of Medical Personnel on Chemotherapy for Gastric and Colorectal Cancer]. Gan to kagaku ryoho Cancer \& chemotherapy 2018, 45(8):1171-1176.

13. Ferreira JA, Magalhães A, Gomes J, Peixoto A, Gaiteiro C, Fernandes E, Santos LL, Reis CA: Protein glycosylation in gastric and colorectal cancers: Toward cancer detection and targeted therapeutics. Cancer Lett 2017, 387:32-45.

14. Strong VE, Wu AW, Selby LV, Gonen M, Hsu M, Song KY, Park CH, Coit DG, Ji JF, Brennan MF: Differences in gastric cancer survival between the U.S. and China. J Surg Oncol 2015, 112(1):31-37.

15. Li M, Wan X, Wang Y, Sun Y, Yang G, Wang L: Time trends of esophageal and gastric cancer mortality in China, 1991-2009: an age-period-cohort analysis. Sci Rep 2017, 7(1):6797. 
16. Zhang KC, Chen L: [Emphasis on standardization of minimally invasive surgery for gastric cancer]. Zhonghua wai ke za zhi [Chinese journal of surgery] 2018, 56(4):262-264.

17. AM DAS: Multiorganic resections in gastric cancer. Revista do Colegio Brasileiro de Cirurgioes 2017, 44(6):549-552.

18. Tan Z: Recent Advances in the Surgical Treatment of Advanced Gastric Cancer: A Review. Medical science monitor : international medical journal of experimental and clinical research 2019, 25:35373541.

19. Liu H, Liu Y, Bian Z, Zhang J, Zhang R, Chen X, Huang Y, Wang Y, Zhu J: Circular RNA YAP1 inhibits the proliferation and invasion of gastric cancer cells by regulating the miR-367-5p/p27 (Kip1) axis. Molecular cancer 2018, 17(1):151.

20. Yasui W, Oue N, Aung PP, Matsumura S, Shutoh M, Nakayama H: Molecular-pathological prognostic factors of gastric cancer: a review. Gastric cancer : official journal of the International Gastric Cancer Association and the Japanese Gastric Cancer Association 2005, 8(2):86-94.

21. Gao HY, Huo FC, Wang HY, Pei DS: MicroRNA-9 inhibits the gastric cancer cell proliferation by targeting TNFAIP8. Cell proliferation 2017, 50(2).

22. Liu T, Xia B, Lu Y, Xu Y, Lou G: TNFAIP8 overexpression is associated with platinum resistance in epithelial ovarian cancers with optimal cytoreduction. Hum Pathol 2014, 45(6):1251-1257.

23. Xing Y, Liu Y, Liu T, Meng Q, Lu H, Liu W, Hu J, Li C, Cao M, Yan S et al: TNFAIP8 promotes the proliferation and cisplatin chemoresistance of non-small cell lung cancer through MDM2/p53 pathway. Cell communication and signaling : CCS2018, 16(1):43.

24. Fricker M, Tolkovsky AM, Borutaite V, Coleman M, Brown GC: Neuronal Cell Death. Physiological reviews 2018, 98(2):813-880.

25. Bialik S, Dasari SK: Autophagy-dependent cell death - where, how and why a cell eats itself to death. 2018, 131(18).

26. Chen Y, Sun Z, Qi M, Wang X, Zhang W: INPP4B restrains cell proliferation and metastasis via regulation of the PI3K/AKT/SGK pathway. 2018, 22(5):2935-2943.

27. Xia $\mathrm{P}, \mathrm{Xu} X Y$ : PI3K/Akt/mTOR signaling pathway in cancer stem cells: from basic research to clinical application. American journal of cancer research 2015, 5(5):1602-1609.

28. Tian L, Zhao Z, Xie L, Zhu J: MiR-361-5p suppresses chemoresistance of gastric cancer cells by targeting FOXM1 via the PI3K/Akt/mTOR pathway. Oncotarget 2018, 9(4):4886-4896.

29. Liu F, Zhang Y, Men T, Jiang X, Yang C, Li H, Wei X, Yan D, Feng G, Yang J et al: Quantitative proteomic analysis of gastric cancer tissue reveals novel proteins in platelet-derived growth factor $b$ signaling pathway. Oncotarget 2017, 8(13):22059-22075.

30. Jin S, Zhang X, Miao Y, Liang P, Zhu K, She Y, Wu Y, Liu DA, Huang J, Ren J et al: m(6)A RNA modification controls autophagy through upregulating ULK1 protein abundance. Cell Res 2018, 28(9):955-957. 
Figures

Figure.1

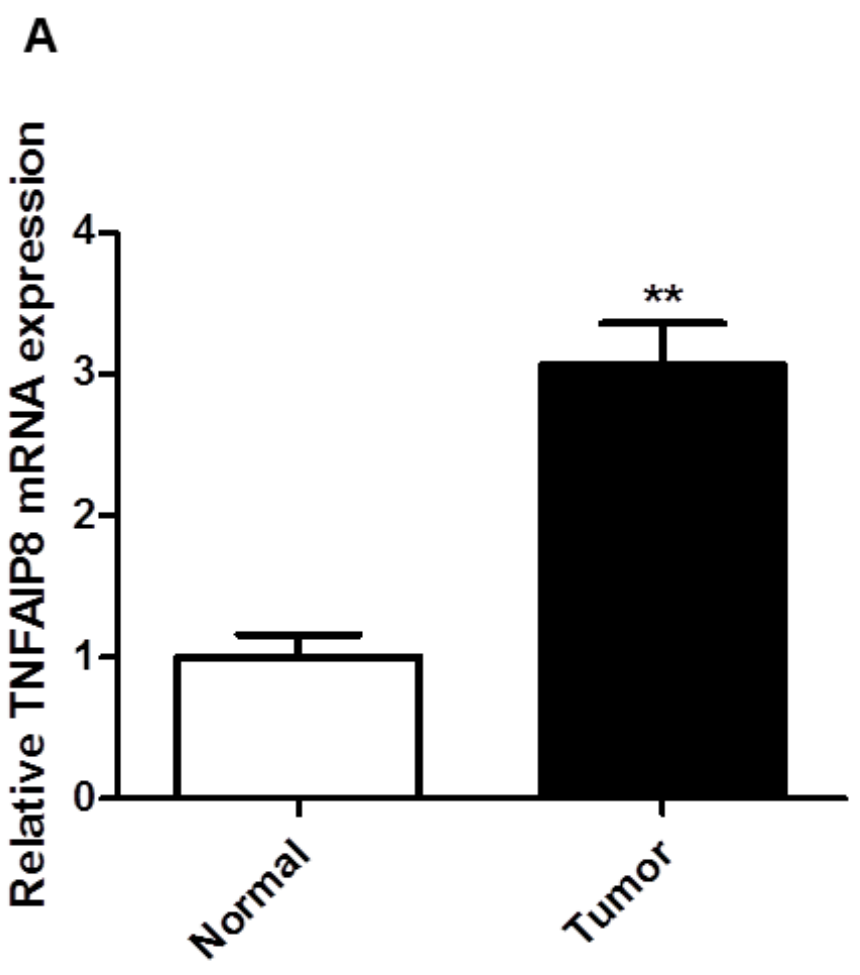

B

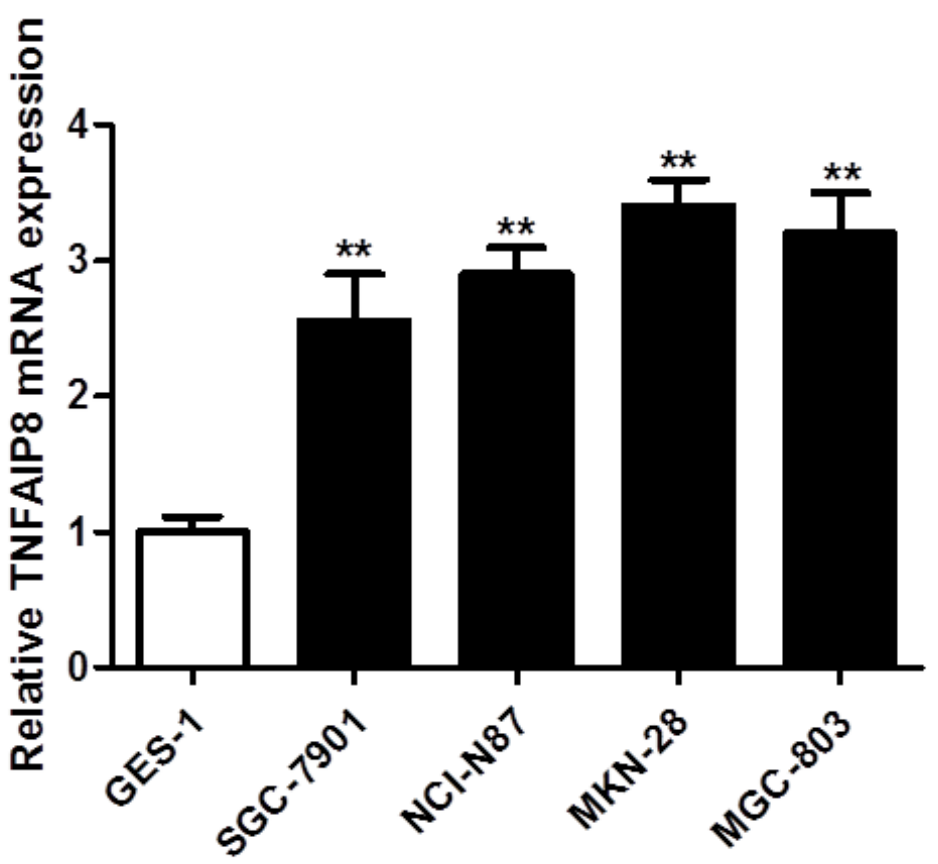

Figure 1

TNFAIP8 gene expression in gastric mucosal cells and four types of gastric cancer cells Data are expressed as the mean \pm SD $(n=8)$. Compared with normal or GES-1: ${ }^{*} p<0.05$ and ${ }^{* *} p<0.01$ 
Figure.2

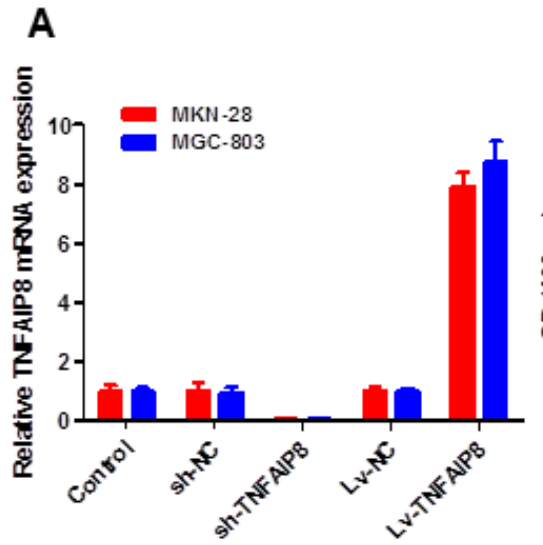

C
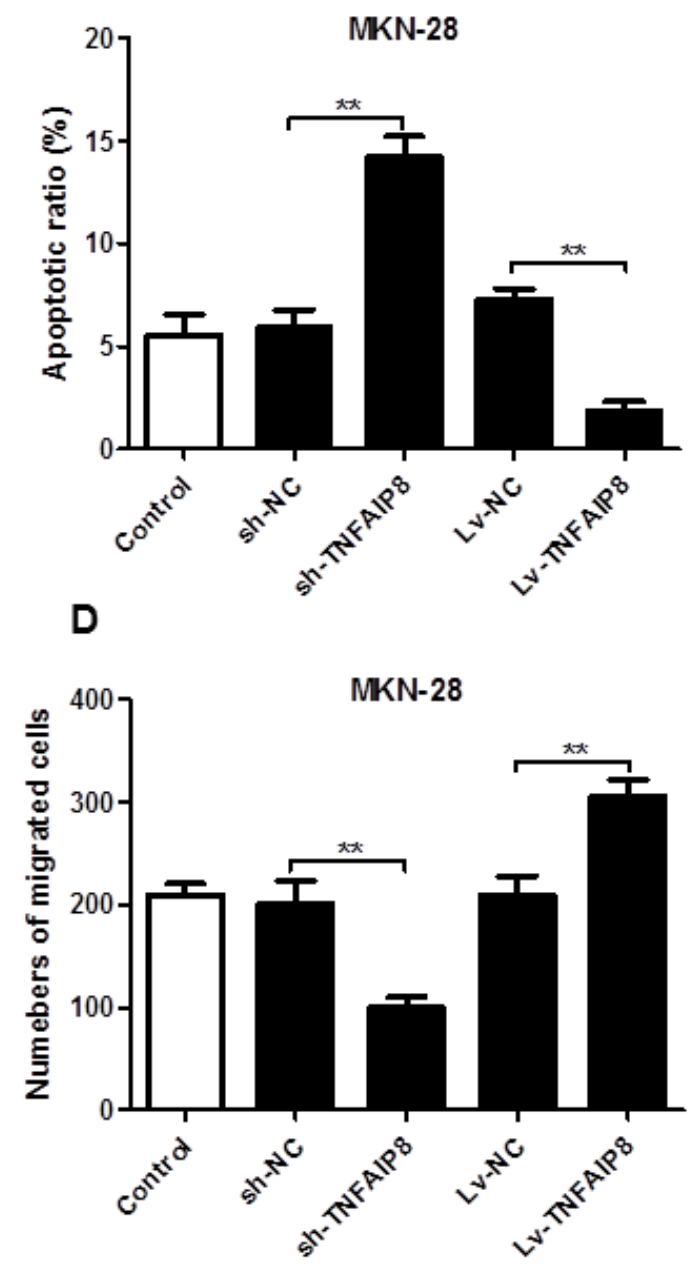

MKN-28
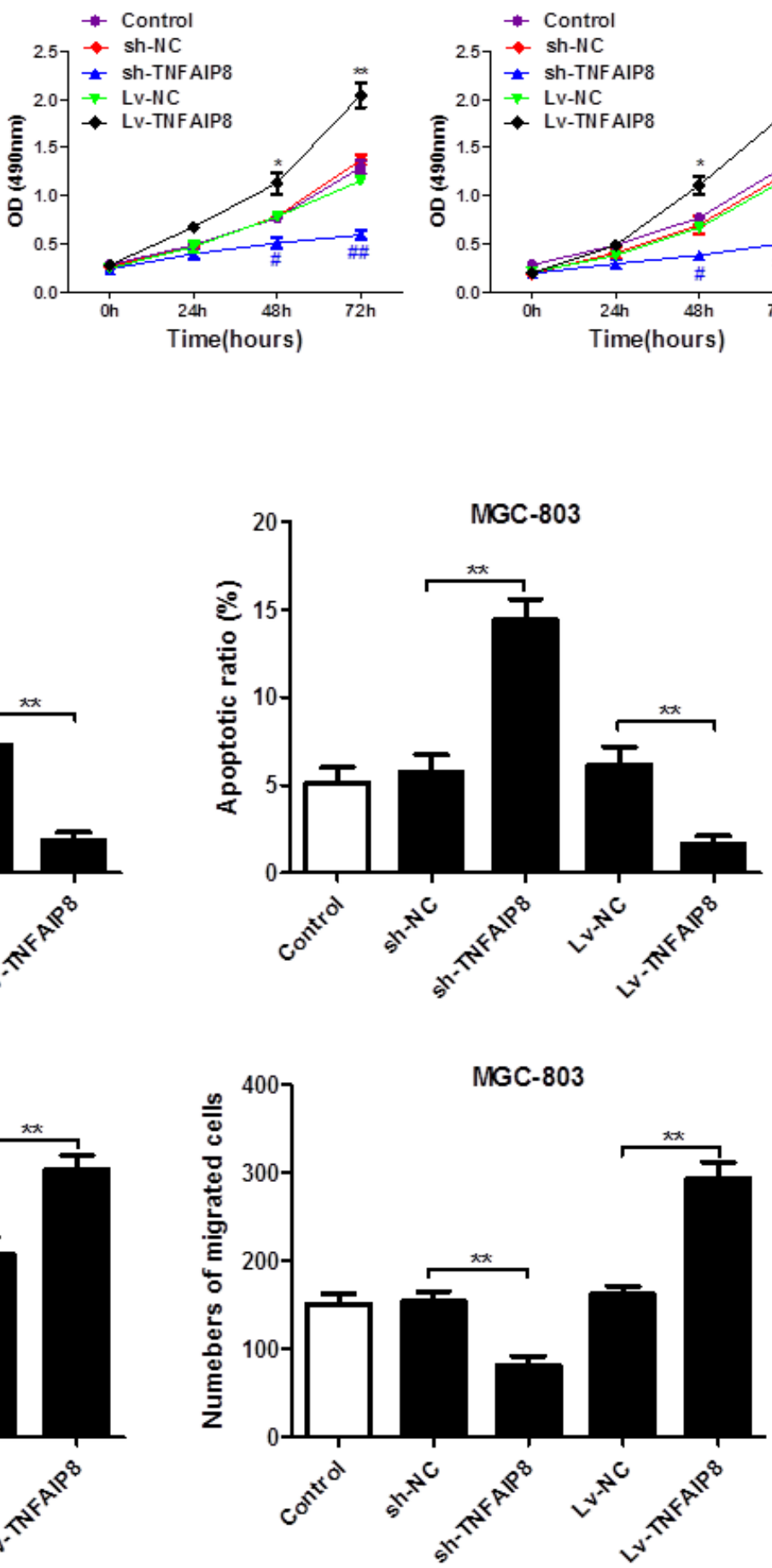

Figure 2

Overexpression of TNFAIP8 gene increased cell viability, reduced apoptotic rate and increased migration ability Data are expressed as the mean $\pm S D(n=8)$. Compared with control: ${ }^{*} p<0.05$ and ${ }^{* \star} p<0.01$ 
Figure.3

A

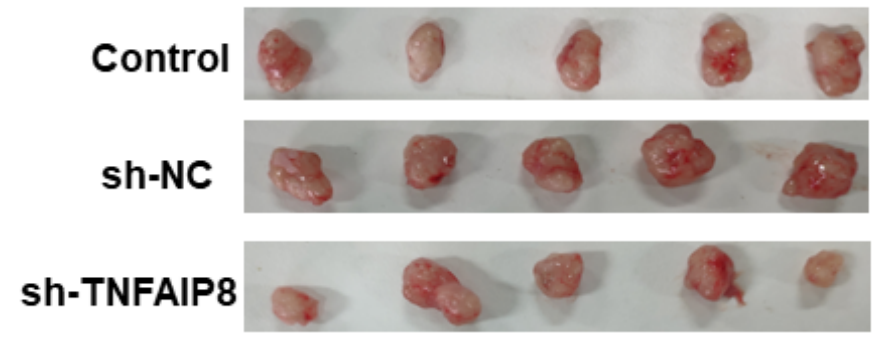

Lv-NC

LV-TNFAIP8

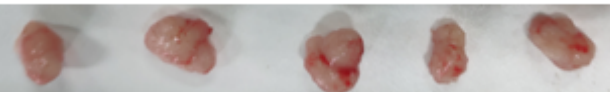

C

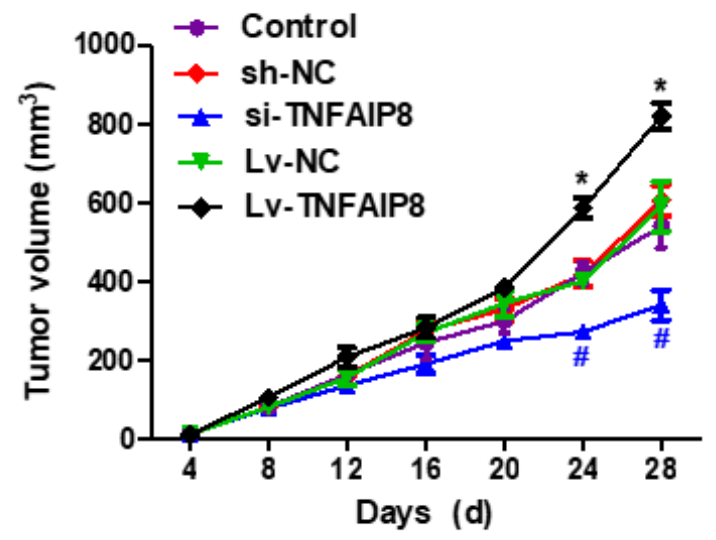

B
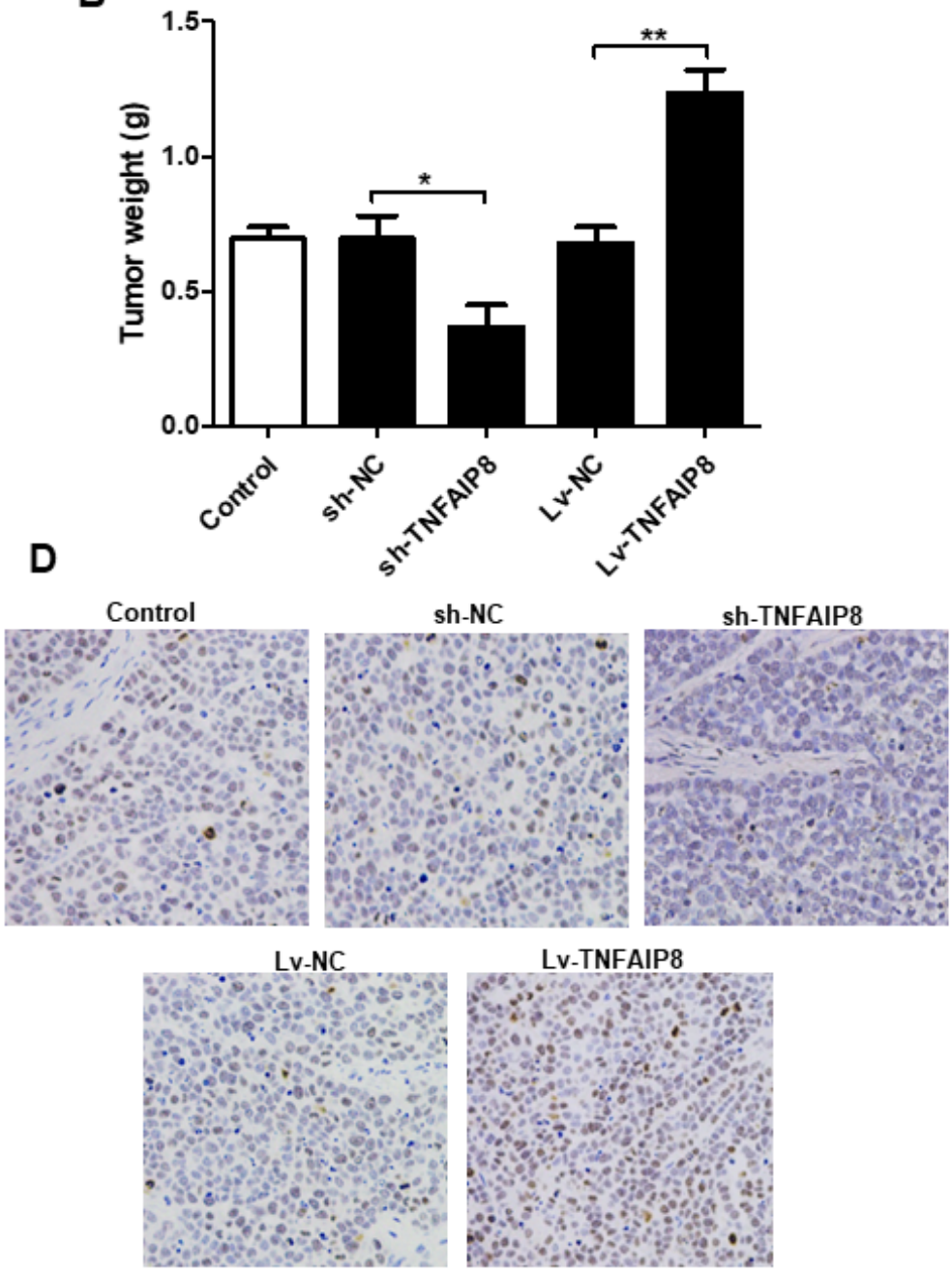

Lv-TNFAIP8

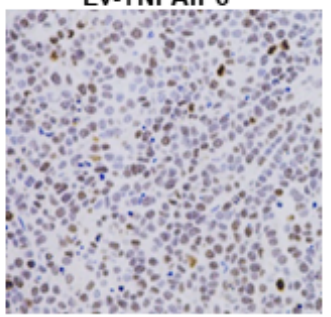

Figure 3

Overexpression of TNFAIP8 gene increased tumor volume and tumor weight in transplanted tumor mice Data are expressed as the mean \pm SD $(n=8)$. Compared with LV-NC or Sh-NC: ${ }^{*} p<0.05$ and ${ }^{* \star} p<0.01$ 
Figure.4

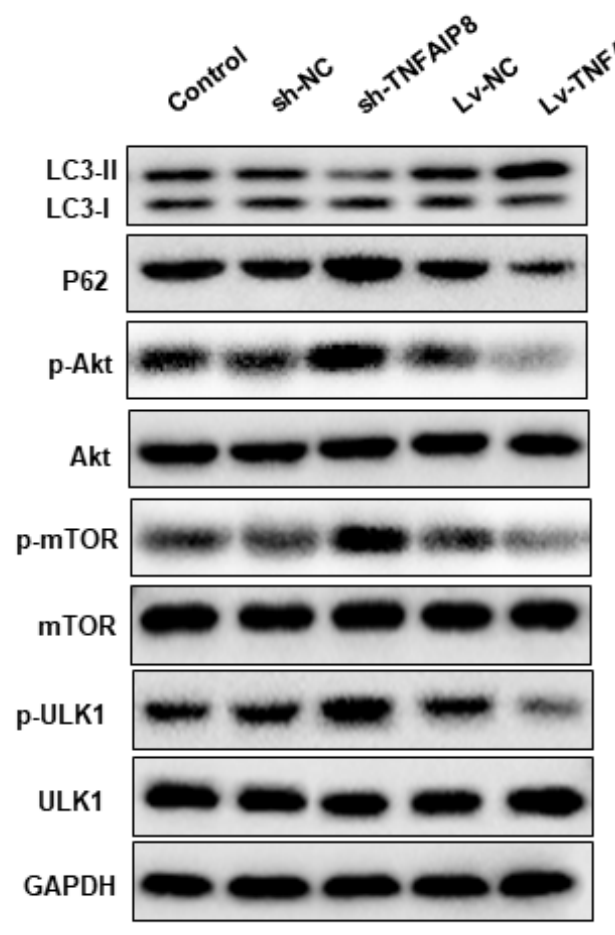

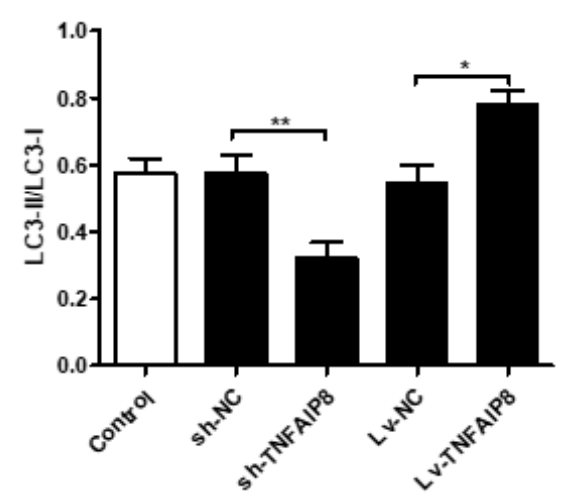
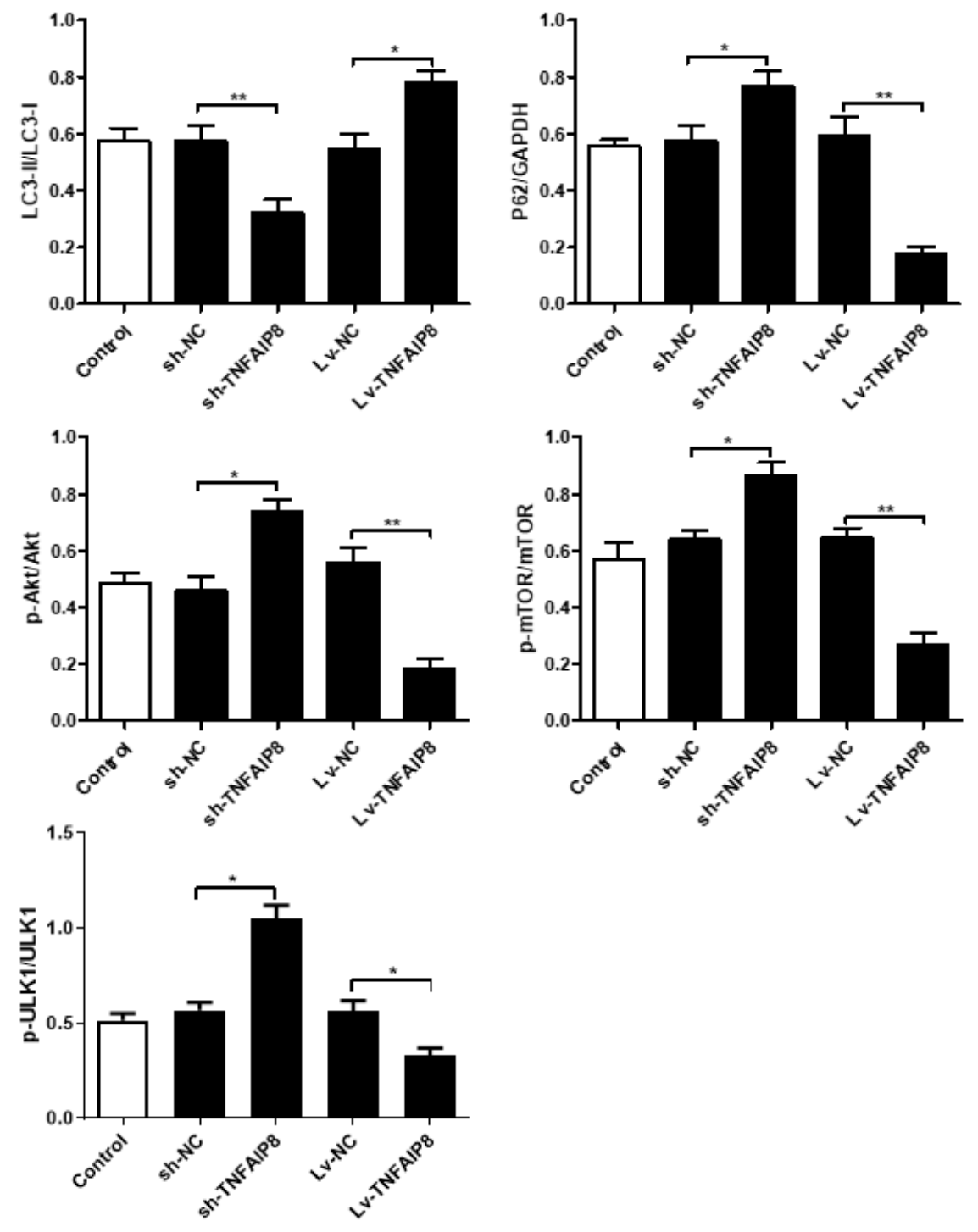

Figure 4

Overexpression of TNFAIP8 gene activated autophagy and inhibited mTOR-Akt-ULK1 signaling pathway Data are expressed as the mean \pm SD $(n=8)$. Compared with LV-NC or Sh-NC: ${ }^{*} p<0.05$ and ${ }^{* *} p<0.01$ 
Figure.5

A

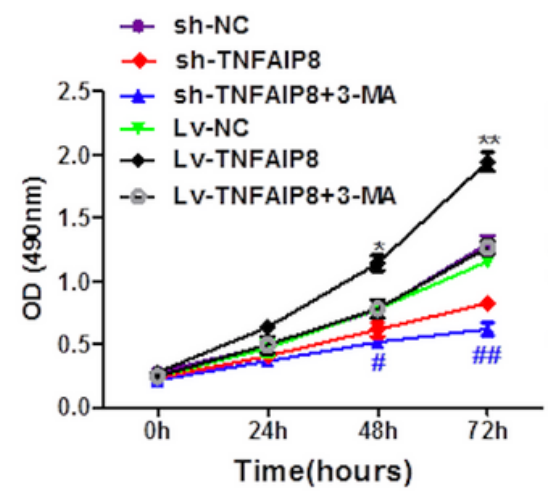

B

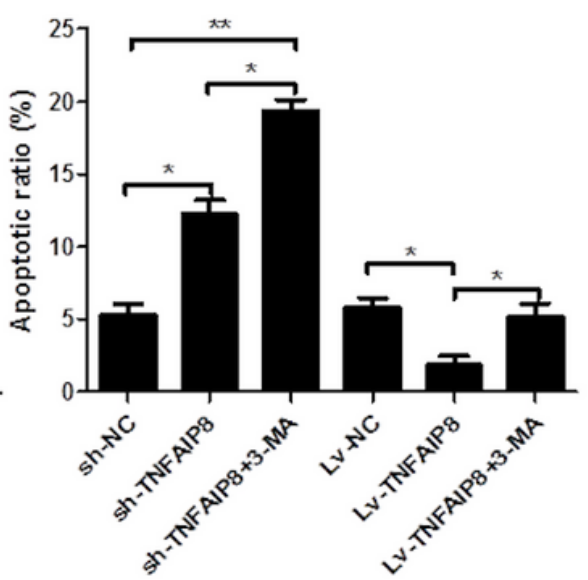

C

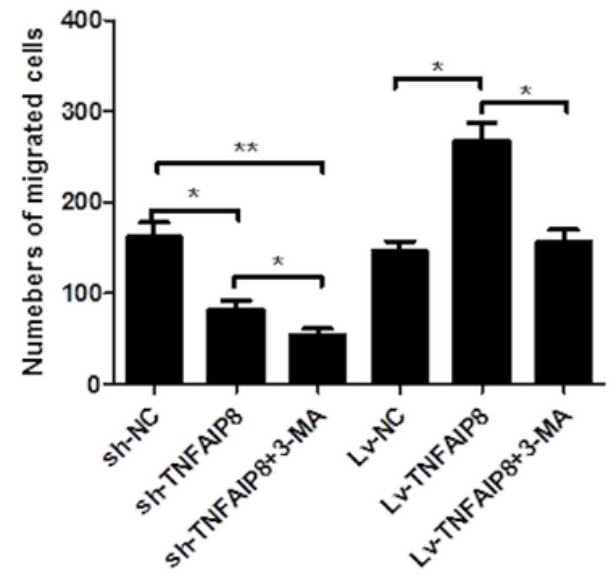

\section{Figure 5}

Autophagy inhibitor and activator demonstrated the role of TNFAIP8 genes in regulating autophagy signals Data are expressed as the mean \pm SD $(n=8)$. Compared with LV-NC or Sh-NC: ${ }^{*} p<0.05$ and $* * p<$ 0.01 
Figure.6
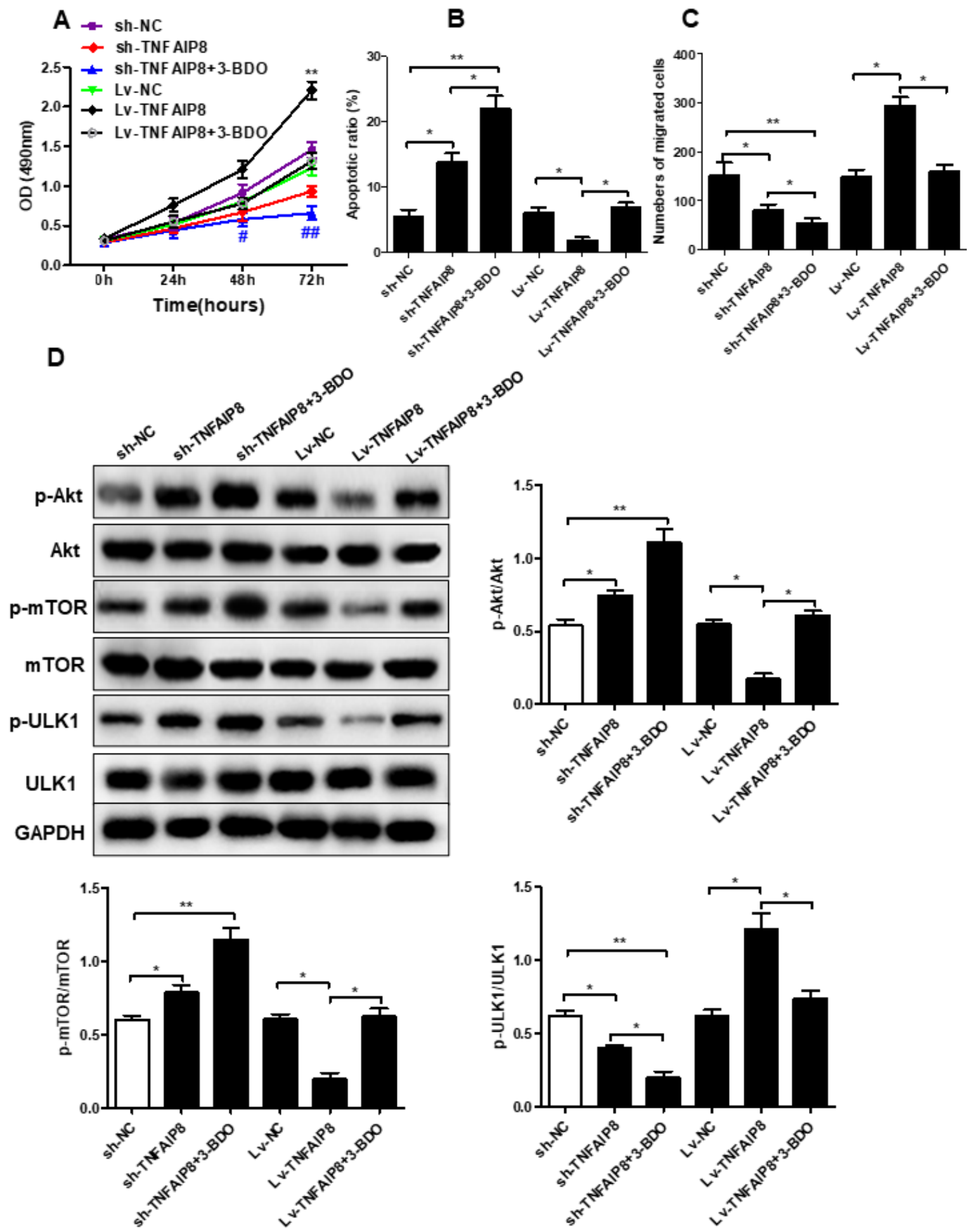

Figure 6

Autophagy activator demonstrated the role of TNFAIP8 genes in regulating autophagy signals Data are expressed as the mean \pm SD $(n=8)$. Compared with LV-NC or Sh-NC: ${ }^{*} p<0.05$ and ${ }^{* \star} p<0.01$ 